\title{
Crossover Operators in Genetic Algorithms: A Review
}

\author{
Padmavathi Kora \\ Gokaraju Rangaraju \\ Institute of Engineering \& \\ Technology, Hyderabad
}

\author{
Priyanka Yadlapalli \\ Gokaraju Rangaraju \\ Institute of Engineering \& \\ Technology, Hyderabad
}

\begin{abstract}
Genetic Algorithms are the population based search and optimization technique that mimic the process of natural evolution. Genetic algorithms are very effective way of finding a very effective way of quickly finding a reasonable solution to a complex problem. Performance of genetic algorithms mainly depends on type of genetic operators which involve crossover and mutation operators. Different crossover and mutation operators exist to solve the problem that involves large population size. Example of such a problem is travelling sales man problem, which is having a large set of solution. In this paper we will discuss different crossover operators that help in solving the problem.
\end{abstract}

\section{Keywords}

Genetic Algorithm; Mutation; crossover; Selection; travelling salesman problem

\section{INTRODUCTION}

Evolutionary algorithms always follow the Darwin concept of "Survival of the fittest" which is extensively used for optimization problems for nearly four decades (J. Holand et.al, 1975), (Padmavathi Kora et.al, 2016) (Ambika Annavarapu et.al, 2016). Evolutionary algorithms are classic examples of heuristic search algorithms yet they do not yield exact optimal solutions, but will certainly help to find better optimal solutions when compared to their parallel counterparts within less amount of time. Genetic algorithms and Evolutionary programming combined provide an excellent system for effectively sampling large search areas with basic technique is widely applicable. Genetic algorithms are by nature have adaptive optimization algorithms which resemble the process of natural selection and genetics (D.E. Goldberg et.al, 1989).Both exploitation and exploration techniques are by and large accountable for the performance of genetic algorithms. Exploitation in fact is defined as usage of already existing information to find out the better solution and Exploration means to find out a novel and unknown solution in investigation space. The crux of the genetic algorithms emerges from the capability to combine both exploration and exploitation in an ideal way. Biological genetics model is regarded as one of the best role model of genetic algorithm. A generic genetic algorithm comprises of the following operations namely: Initialization, Selection, Reproduction and Replacement. Initialization represents creation of initial population by utilizing suitable encoding scheme. Selection operator chooses the individuals arbitrarily or chosen according to their fitness. Crossover and mutation genetic operators are exclusively involved to strike balance between exploitation and exploration. During replacement, the old ones are succeeded by new offspring's (Manju Sharma et.al, 2013). By definition, crossover selects genes from parent chromosomes and creates a new offspring. The simplest way is to choose randomly some crossover point, point copy from a first parent and then everything after a crossover point copy from the second parent. In genetic algorithms, chromosomes denoted as linear strings of symbols (C Ryan et.al, 2000).

It is very important that in order to enhance the functionality of genetic algorithm problem there is a specific requisition of crossover. After reproduction or after the so called selection process, the population comprises of better individuals. Reproduction or selection produces same individual but the progeny are improved when compared to the older ones. The main feature of genetic algorithms is to merge both exploration and exploitation in an ideal way. In reality, we are accustomed to the fact that it is the population size that has an impact on the performance of genetic algorithm which results in the problem of genetic drift which is rampant mostly in the case of multimodal search space. By initiating a local search method within the genetic operators we can produce new genes that can resolve the problem of genetic drift and accelerate the search towards global optima (W.E. Hart et.al, 1994). When a genetic algorithm with a local search method is combined a hybrid genetic algorithm/mimetic algorithm is evolved. Some crossover operators are utilized for exploitation as well as for exploration. The main emphasis of this paper is to study various types of crossover operators

\section{TRAVELLING SALESMAN PROBLEM}

The Travelling Salesman Problem (TSP) is one of the most widely used and well studied for combinational optimization problems. The statement of Travelling Salesman Problem (TSP) was made by Karl Menger in 1930 whose statement is very uncomplicated, but it is one of the most challenging and popular problems in the field of Operational Research. Large number of articles and research papers have been written and published on this problem. The most common practical interpretation of TSP is that a salesman seeking the shortest tour through $\mathrm{n}$ clients or cities. The Travelling Salesman Problem is one of the famous NP-hard problems, which means that there is no perfect algorithm to solve it in polynomial time. The minimal time required to obtain optimal solution is exponential (Ivan Brezina Jr et.al, 2011). The main motive is to find the shortest path or the path with least cost. It is very complicated as starting and finishing points are same and we have to return to the original point after visiting each of the vertex only once. The most prevalent practical applications of TSP is that it not only involves regular distribution of goods or resources, but finding out the shortest path for costumer servicing route, planning bus routes, vehicle routing, computer wiring, machine sequencing and scheduling, frequency assignment in communication networks, but also in the areas that have nothing to do with travel routes. 


\section{VARIOUS METHODS USED FOR RESOLVING TSP}

In 1997, Rong Yang initiated several knowledgeaugmented genetic operators that guide the genetic algorithm more directly towards better quality of the population but not trapped in local optima prematurely. The algorithm uses an avid crossover operator along with two advanced mutation operations based on the 2-opt and 3-opt heuristics (Rong Yang et.al, 1997). In 2001, Chiung Moon introduced the concept of topological sort (TS), which is as an or interpreted as ordering of vertices in a directed graph. Moreover, a new crossover operation is introduced for the proposed GA (Chiung Moon et.al, 2001). In 2004, new knowledge based multiple inversion operators and neighborhood swapping operator is proposed by Shubhra Sankar Ray (ShubraShankar et.al, 2001). In 2005, Lawrence V. Snyder gave a heuristic to resolve the generalized travelling salesman problem. The approach incorporates a local tour development heuristic into a randomkey genetic algorithm. The algorithm was very much successful when tested on a set of 41 standard problems with familiar optimal objective values. In 2005, Milena Karova found that genetic algorithm when implemented gives a maximal approximation of the problem, thus reforming the acquired solution with genetic operators. In the year 2006, Plamenka Borovska analyzed the efficiency of parallel computation of travelling salesman problem applying the genetic approach on a casual multicomputer cluster .In 2007, a two-level genetic algorithm (TLGA) was developed for a case, with special treatment consisting of neither intracluster paths nor inter- cluster paths, thus realizing an integrated evolutionary optimization for both levels of the CTSP. In 2008, a software system is developed to determine the ideal route for Travelling Salesman Problem by employing Genetic Algorithm technique. In 2009, S.N. Sivanandam presented two approaches i.e Genetic Algorithms and Particle swarm optimization in order to find a solution to the given objective function by applying different procedures and computational techniques; as a result of which their performance can be evaluated and compared. In 2016, Hybrid Particle Swarm Optimization (PSO) and Bacterial foraging algorithm has been initiated for the classification of ECG.

\section{GA METHODOLOGY}

A genetic algorithm defines how an algorithm can generate a modified copy of itself in order to achieve an objective. The progeny would match against the criteria to ensure that the objective would be eventually attained. Genetic algorithm which is inspired by Darwin's theory is all about evolution. It is not that difficult to program or realize, since they are biological based.

\section{TYPES OF CROSSOVER OPERATORS \\ 1) Single Point Crossover}

Single point crossover is the most approved crossover which is widely in use. A crossover site is randomly selected along the length of the mated strings and bits which are very next to the cross-sites are interchanged. When suitable site is chosen then better progeny can be obtained by combining the good qualities of the parents. If appropriate site is chosen, better offspring can be obtained by combining good quality parents else it harshly hampers string quality. In single point crossover the head and tail of one chromosome break up and if both head and tail have good genetic material then none of the offspring will get the both good features directly.

\section{2) N Point Crossover}

The N-point crossover was first implemented by De Jong in 1975. It has many cross over sites but rule used is same as we used in single point crossover. In 2-point crossover significance of crossover sites is 2 . Adding more and more crossover sites effects the disruptions of building blocks that sometimes reduce the performance of genetic algorithm. But it allows the head and tail section of a chromosome to be accepted together in the offspring

\section{3) Uniform Crossover}

Uniform crossover do not fragments the chromosomes for recombination. Each gene in offspring is created by copying it from the parent chosen according to the corresponding bit in the binary crossover mask of same length as the length of the parent chromosomes. If the bit in crossover mask is 1 , then the resultant gene is copied from the first parent and if the bit in crossover mask is 0 , then the resultant gene is copied from the second parent. A new crossover mask is generated arbitrarily for each pair of parent chromosomes. The quantity of crossover point is not fixed initially. So, the offspring have a mixture of genes from both the parents

\section{4) Three Parent Crossover}

In this crossover, three parents are chosen arbitrarily. Each gene of the 1st parent is compared with the equivalent gene of the 2 nd parent. If both genes are similar, the gene is occupied for offspring or else the equivalent gene from the 3 rd parent is taken for the offspring. It is mostly used in case of binary encoded chromosomes.

\section{5) Arithmetic Crossover}

Arithmetic crossover is used in case of real-value encoding. Arithmetic crossover operator linearly combines the two parent chromosomes.Two chromosomes are particular randomly for crossover and create two offspring's which are linear mixture of their parents

\section{6) Partially mapped Crossover}

Partially Matched or Mapped Crossover (PMX) is the most frequently used crossover operator. It was proposed by Goldberg and Lingle for Travelling Salesman Problem In Partially Matched Crossover, two chromosomes are associated and two crossover sites are chosen arbitrarily. The fraction of chromosomes between the two crossover points gives a corresponding selection that undergoes the crossover process through position-by- position exchange operations (Padmavathi Kora et.al, 2015). PMX tends to respect the absolute positions

\section{7) Crossover ORDER $(O X)$}

It was proposed by Davis and also used for chromosomes with permutation encoding. The process starts in a way similar to that of PMX by choosing two crossover points. But in its place of using point-by-point exchanges as in case of PMX, order crossover applies sliding motion to fill up the left out holes by sending the mapped positions. It copies the portion of permutation elements between the crossover points from the cut string directly to the offspring, insertion them in the same absolute position (Padmavathi Kora et.al, 2015). OX tends to respect the relative positions.

\section{8) Cycle Crossover (CX)}

This crossover is used for chromosomes with permutation encoding. During recombination in cyclic crossover there is a limitation that each gene either comes from the one parent or the other. The fundamental model at the back cycle crossover is that each allele comes from one parent jointly with its 
position. To construct a cycle of alleles from parent1 we have to start with the first allele of parent1. Then look at the allele at the equal position in parent 2 and go to the position with the same allele in Parent1.insert this allele to the cycle and do again above step until you reach your destination at the first allele of parent1. Put the alleles of the cycle in the first child on the positions they have in the first parent and the remaining alleles of first child come from the second parent along with their position. Produce next cycle from parent 2 .

\section{SOLVING TSP USING GENETIC ALGORITHM}

A genetic algorithm is popular to find a solution of a problem in much less time. Even though it might not find the most excellent solution of the problem but it can help in finding a near ideal solution for a 100 city tour in less than a minute. The following are some fundamental steps of our proposed work

Encoding: Permutation encoding is most popular way to solve the TSP. We characterize cities with an integer value, and after that we initialize the population.

- Distance matrix: distance matrix is a representation of $N \times N$ matrix of point to point distances.

- Selection based on fitness function: the fitness function will be total expenditure of the visit represented by each chromosome. The smaller the sum, the fitter the solution represented by that chromosome.

- Generating arbitrary numbers equal to population size. Best of two routes will be chosen using tournament selection to relate Mutation.

- Next generation of population size will be produced. Process will go through predefined iterations.

- After the final iteration the smallest number of distance size will be displayed as result.

\section{CONCLUSION}

In this paper, we have discussed eight types of mutations strategy in the genetic algorithm procedure to create new and better generations. As we learnt that genetic algorithms appear to be a good solution for TSP, however it very much depends on the technique the difficulty is encoded and which crossover and mutation methods are used. So our labors will decide which mutation operator will always gives end result to undertake most of the real life applications of travelling salesman problem.

\section{FUTURE SCOPE}

It appears that major dilemma with the genetic algorithm for TSP is that to preserve the formation from the parent's chromosomes and still finish up with a permissible tour in the child chromosomes. Possibly a improved crossover or mutation routine that remain structure from parent chromosome would give a far better clarification than we have previously establish for some TSP. As a potential work we want to prolong the same process with different other crossover and mutation operator to give an improved solution to a TSP.

\section{REFERENCES}

[1] Ambika Annavarapu, Padmavathi Kora, "ECG-based Atrial Fibrillation detection using different orderings of Conjugate Symmetric-Complex Hadamard Transform," International Journal of Cardiovascular Academy, Elsevier, Aug 2016.

[2] C. Ryan, Automatic re-engineering of software using genetic programming, Genetic Programming Series. Kluwer Academic Publishers, ISBN 0-7923-8653-1, 2000.

[3] Chiung Moon, Jongsoo Kim, GyunghyunChoi ,YoonhoSeo," An efficient genetic algorithm for the traveling salesman problem with precedence constraints", European Journal of Operational Research 140 (2002) 606-617, accepted 28 February 2001

[4] D.E. Goldberg, Genetic algorithms in search, optimisation, and machine learning, Addison Wesley Longman, Inc., ISBN 0-201-15767-5, 1989

[5] Ivan Brezina Jr.,ZuzanaCickova, "Solving the Travelling Salesman Problem using the Ant colony Optimization", Management Information Systems, 2011, Vol. (6), No. (4).

[6] J. Holland, Adaptation in natural and artificial systems, University of Michigan Press, Ann Arbor, 1975.

[7] Manju Sharma , Novel Knowledge based Selective Tabu Initialization in Genetic algorithm, IJARCSSE, Volume 3, Issue 5, May 2013

[8] Padmavathi Kora, K. Sri Rama Krishna, "Bundle Block Detection using Genetic Neural Network," International System design and Intelligent Applications (INDIA), AISC, Springer, Jan 2016.

[9] Padmavathi Kora, and Sri Rama Krishna, "Hybrid Bacterial Foraging and Particle Swarm Optimization for detecting Bundle Branch Block," SpringerPlus, Springer, vol 4, no 1, 481, Sep 2015.

[10] Padmavathi Kora, and Sri Ramakrishna Kalva, "Improved Bat algorithm for the detection of myocardial infarction," SpringerPlus, Springer, vol 4, no. 1, pp. 1-18, Nov 2015.

[11] Rong Yang, "Solving Large Travelling Salesman Problems with Small Populations". IEEE 1997.

[12] ShubhraSankar Ray, Sanghamitra Bandyopadhyay and Sankar K.Pal," New Operators of Genetic Algorithms for Traveling Salesman Problem", 2000IEEE.

[13] W. E. Hart, "Adaptive global optimization with local search, Doctoral diss.", San Diego, University of California, 1994. 